\title{
Peripheral nerve blockade as an exclusive approach to obturator nerve block in anterior cruciate ligament reconstructive surgery
}

\author{
Marina Simeoforidou ${ }^{1}$, Meraxia Bareka ${ }^{1}$, George Basdekis ${ }^{2}$, Katerina Tsiaka ${ }^{1}$, Eleni Chantzi ${ }^{1}$, and \\ George Vretzakis ${ }^{1}$
}

Departments of ${ }^{1}$ Anesthesiology Clinic, ${ }^{2}$ Orthopedic Clinic, University Hospital of Larissa, Trikala, Greece

Background: Obturator nerve block plays an additive role on the quality of analgesia for knee surgery. Since the use of dual guidance increases the success rate of nerve blocks, we investigated the feasibility of performing anterior cruciate ligament reconstruction under dual-guided blockade of obturator with femoral and sciatic nerves. Furthermore, we propose a novel method for the assessment of obturator nerve block.

Methods: Fifty-seven patients undergoing anterior cruciate ligament repair were studied. Neurostimulating needles were guided out-of-plane by ultrasound. To induce the obturator nerve block, $10 \mathrm{ml}$ of ropivacaine $0.5 \%$ were injected after eliciting contractions of adductor longus, brevis and magnus followed by block assessment for 30 minutes by examining the patient lift and left down the leg.

Results: The sonographic recognition of obturator nerve was easy and quick in all cases. Time for applying the block was $119.9 \pm 79.2 \mathrm{sec}$. Assessing this block with lifting-leaving down the leg gave satisfactory results in $24.0 \pm 5.07 \mathrm{~min}$. After performing femoral-sciatic blocks, the inflation of tourniquet resulted in VAS score of $>0$ in 2/57 patients and operation in $12 / 57$. Total dose of fentanyl was $120.1 \pm 64.6 \mu \mathrm{g}$ and of midazolam $1.86 \pm 0.8 \mathrm{mg}$. In 6 patients propofol was administered for sedation and 1 of them required ventilation with laryngeal mask airway, converting the anesthesia technique to general anesthesia.

Conclusions: Our data suggest that anterior cruciate ligament reconstruction can be performed under obturator-femoral-sciatic blocks. Identification of obturator nerve with ultrasound is easy and the block can be assessed by observing how the patient lifts and leaves down the leg. (Korean J Anesthesiol 2013; 65: 410-417)

Key Words: Anterior cruciate ligament, Nerve block, Neurostimulation, Obturator nerve, Ultrasonography.

Received: March 25, 2013. Revised: April 20, 2013. Accepted: April 29, 2013.

Corresponding author: Meraxia Bareka, M.D., Department of Anesthesiology Clinic, University Hospital of Larissa, Mezourlo, Larissa 41110, Greece. Tel: 00306947845083, Fax: 00302413502952, E-mail: barekametaxia@hotmail.com

(c) This is an open-access article distributed under the terms of the Creative Commons Attribution Non-Commercial License (http:// creativecommons.org/licenses/by-nc/3.0/), which permits unrestricted non-commercial use, distribution, and reproduction in any medium, provided the original work is properly cited. 


\section{Introduction}

Anterior cruciate ligament (ACL) reconstructive surgery can be performed under a variety of anesthetic techniques including peripheral nerve blockade. For example, lumbar plexus, the psoas compartment block is a useful but controversial technique, due its relatively high rates of failure and serious complications $[1,2]$. Theoretically, an alternative method to perform ACL reconstructive surgery is by blocking all the three, obturator, femoral and sciatic nerves more peripherally as they innervate the anatomic area and the structures in concern. Sensory branches arising from the obturator nerve, such as the articular branch for the knee and the cutaneous branches of the medial thigh, contribute to the sensation of pain during knee surgery. The contribution of obturator nerve block to the quality of analgesia for major knee surgeries has been recognized in studies demonstrating that patients experienced significantly lower pain scores and showed reduced opioid requirements perioperatively when an obturator nerve block was added [3]. Recently, in a study testing an ultrasound-guided technique for obturator nerve block, the patients were operated for ACL reconstruction under a sciaticfemoral block combined with obturator block and completed surgery without requiring general anesthesia and with only a $14 \%$ of patients needed a small-dose opioid supplementation [4]. Techniques for blocking the femoral and subgluteal sciatic nerve with the use of a nerve stimulation or under dual guidance have been reported with satisfactory results. In contrast, even with ultrasound guidance, due to obturator's nerve high degree of variability [5,6], dual guidance needs careful attention. The intrafascial course of the obturator nerve in the thigh and the variability and complicated branching patterns of its divisions and subdivisions challenge the success of the block. Landmarkbased techniques with the use of paresthesia elicitation or nerve stimulation are associated with unsatisfactory block due to branch recognition and lower success rates [7,8]. Lately, the ultrasonographic appearance of the obturator nerve has been described by a number of investigators in volunteers or in clinical circumstances [4,9-11]. In general, ultrasound guidance allows better nerve identification and in studies utilizing this technique for obturator nerve block the reported success rates were more than $90 \%$. However, in most of the studies the obturator nerve block was aiming to improve postoperative analgesia after knee surgery whereas the surgery was carried out using general anesthesia. In addition, due to the multiple variations of the obturator nerve, there is not an easy, reproducible, and commonly accepted clinical test for assessing the success of the block before surgery.

In this study, we propose an approach for blocking the obturator nerve under dual guidance (ultrasound and nerve stimulation) and a simple method for assessing this block. Furthermore, we examined the feasibility of performing arthroscopic reconstruction of the ACL with autograft under peripheral femoral, sciatic and obturator nerve blocks, all applied individually with ultrasound guidance.

\section{Materials and Methods}

The study was registered at the www.clinicaltrials.gov registration site, (registration number NCT01194505) and was conducted prospectively in a tertiary care University Hospital over a 12 month period, after approval from the Institution Ethics committee. Written informed consent was obtained from all patients before entering the study. All recruited patients received standardized anesthesia and intraoperative care by the same specialists (SM, BM). They both had extensive, more than five years experience in peripheral nerve blockade with the use of a nerve stimulator and were certified in the use of ultrasonography. Patients, classified under American Society of Anesthesiologists I and II, aged between 18-45 years, referred for elective, sameday arthroscopic ACL reconstructive surgery using a hamstring autograft including the gracilis tendon were evaluated for inclusion. Exclusion criteria were, patients with serious bleeding disorder, infection at the sites where the blocks were to be applied, diabetes or peripheral neuropathy, neurologic deficits to the affected limb, known allergy to the study drugs, body mass index $(\mathrm{BMI})>35 \mathrm{~kg} / \mathrm{m}^{2}$, psychiatric disorders and communication difficulties.

Intravenous access was established through an $18 \mathrm{G}$ intravenous cannula and the standard monitoring (electrocardiography [ECG], non-invasive blood pressure and $\mathrm{SpO}_{2}$ ) was initiated. Oxygen, at the rate of $5 \mathrm{~L} / \mathrm{min}$ through a facial mask, was also delivered. Light sedation with 1-2 mg midazolam and 50-100 $\mu \mathrm{g}$ fentanyl was established in order to make the patient comfortable without loosing verbal responsiveness during the performance of the peripheral nerve blocks.

Fig. 1 is the flow-chart description of the procedures. Briefly, obturator nerve block was performed under dual guidance, with patients in supine position with the thigh of the affected leg slightly externally rotated. The skin area was sterilized and the ultrasound probe with a linear 5 to $10 \mathrm{MHz}$ transducer (SonoSite, Bothell, WA, USA) was prepared by enclosing it in a sterile sleeve. A sterile 22-gauge $80 \mathrm{~mm}$ insulated block needle connected to a nerve stimulator (Stimuplex; B.Braun, Melsungen, Germany) was also prepared. The inguinal ligament and the adductor longus were palpated. The ultrasound probe was positioned opposite the angle formed by the inguinal crease and adductor longus, with its short diameter approximately $2.5-3.0 \mathrm{~cm}$ in the course of the dichotomous of the angle. It was positioned perpendicular to the skin and the area was scanned with small changes of the angle (Fig. 2). In this position, the pectineus mus- 
cle, the adductor longus, adductor brevis and part of adductor magnus muscles are visible, and the anterior division of the obturator nerve may be recognizable between adductor longus and adductor brevis, while the posterior division may be seen below adductor brevis. In such cases, tracing the anterior ramus proximally towards the nerve, in the sonographic triangle formed by pectineus, adductor longus and adductor brevis muscles, the ultrasound probe was tilted cephalically and the obturator nerve was apparent just as it bifurcated. If the anterior branch was not clearly traceable, the area was scanned with the probe again tilted cephalically from its original position, seeking for a thick hyperechoic image representing nerve tissue and connective tissue. In this plane, next to pectineus and below adductor longus, usually in a depth of approximately $2.0-2.5 \mathrm{~cm}$, a "spider net" image is visualized (Fig. 3). Keeping the probe at the best visualized spot, the skin area of the stimulating needle entry site was anesthetized with $1 \mathrm{ml}$ of $1 \%$ lidocaine. The entry site was located $2.5-3.5 \mathrm{~cm}$ laterally and perpendicularly to the midpoint of the transducer. Then, the needle was inserted through the skin with an out-of-plane approach towards the triangle, aiming

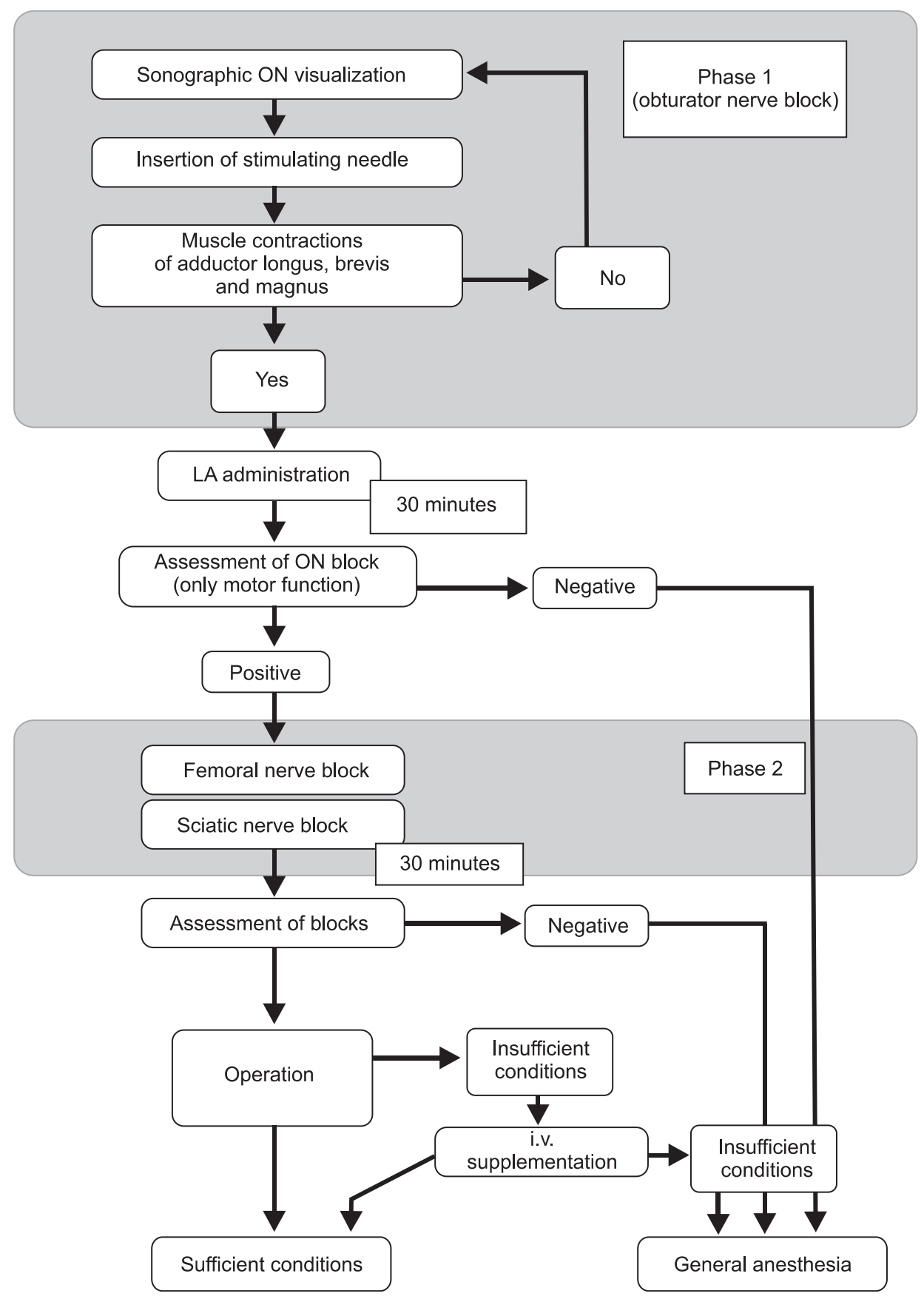

Fig. 1. Diagram showing decisions and actions concerning anesthetic technique. ON: obturator nerve. 


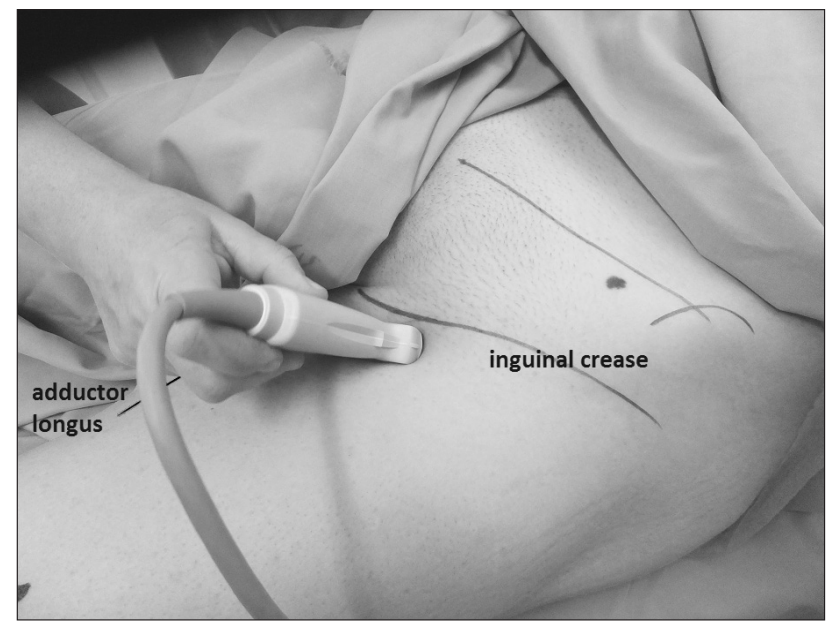

Fig. 2. Position of the ultrasound probe: opposite the angle that is formed by the inguinal crease and adductor longus, with its short diameter in the course of the dichotomous of the angle.

the center of the "spider net". The correct nerve identification was confirmed by elicitation of muscle contractions using the nerve stimulator. The current was set at $0.5 \mathrm{~mA}$ and the stimulation was done without a progressive decrease of the current.

Muscle contractions such as extension of the knee without adduction and internal rotation were considered arising from stimulation of adductor longus and brevis without stimulation of adductor magnus, indicating the identification of the anterior branch. In such a case the needle was directed slightly cephalically. To proceed with the local anesthetic administration it was mandatory that adductor longus, brevis and magnus, all the three muscles, were contracting otherwise the identification attempt was considered negative for obturator nerve and suggestive of branch identification. In this case the above-mentioned steps were repeated. After confirmation of correct needle placement $10 \mathrm{ml}$ of $0.5 \%$ ropivacaine was administered. As stated in Fig. 1, phase 1 represents the procedure for identifying obturator nerve. After $30 \mathrm{~min}$ the success of the block was assessed by evaluating only the motor function. The assessment consisted of a request to the patient to carry out a simple movement which included asking the patient to lift his leg. In a successful obturator block the patient could not lift the leg directly upwards, but he lifted it diagonally outwards (upwards and laterally). Then the patient was asked to leave down the leg. As a result of a successful block the patient put the leg down laterally and could not adduct it to medial line. In case of branch block, lifting and leaving down the leg remains unaffected. By applying this method for assessment we were unable to grade the motor function after the block. The patient can or cannot perform the movement.

After positive assessment for obturator nerve block, patients were given under dual guidance femoral and subgluteal sciatic nerve blocks, with 25 and $10 \mathrm{ml}$ ropivacaine $0.5 \%$ respectively

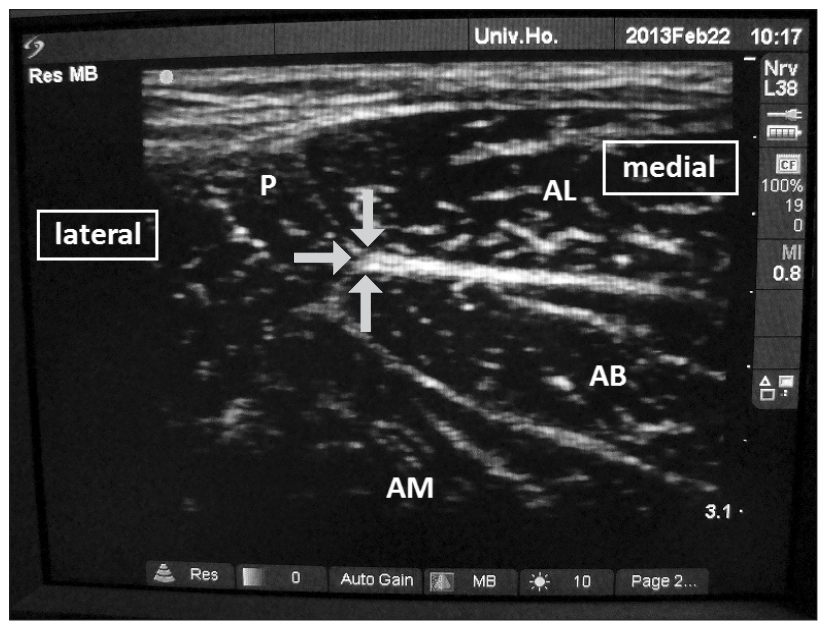

Fig. 3. Ultrasound image where next to pectineus and below adductor longus, a "spider net" image is visualized. Arrows indicate the position of the nerve in the short axis view. P: pectineus, AL: adductor longus, AB: Adductor brevis, AM: adductor magnus.

without any adjunct. The procedures are described in Fig. 1 as phase 2. Sensory and motor blocks were evaluated every 5 min for $30 \mathrm{~min}$ after injection. Sensory blocks were assessed by evaluating the presence or loss of cold-warm feeling and of a sharp sensation with pinprick testing for the femoral in the anterior thigh and for the sciatic in the sole of foot (2 for normal sensory perception, 1 for loss of cold-warm feeling and 0 for loss of pinprick sensation). To evaluate the motor block of the femoral nerve, we examined the ability of the patient to extend the leg against gravity, after the hip was passively flexed at $45^{\circ}$ ( 5 for normal movement, 4 for unable to raise the leg extended against external resistance, 3 for unable to raise the leg extended against gravity, 2 for unable to extend the leg of the operated limb against gravity, after the hip was passively flexed at $45^{\circ}$ by the investigator, 1 for able just to bend knee and 0 for no motion). Sciatic block was evaluated by the motion of the foot and ankle joint ( 3 for normal movement, 2 for unable to push or flex the foot against external resistance, 1 for unable to push or flex the foot against gravity and 0 for no motion).

The patient received paracetamol $1 \mathrm{~g}$ i.v. and parecoxib $40 \mathrm{mg}$ i.v. as part of multimodal analgesia before tourniquet inflation. visual analogue scale (VAS) scores were documented intraoperatively and during the tourniquet inflation and autograft harvesting. The patients were awake and talking with the team and in many instances watching the ACL repair on the screen. Further 1-3 mg midazolam was administered intraoperatively only if the patient was stressed and asked for further sedation. When a patient complained for pain at any point, $50-100 \mu \mathrm{g}$ fentanyl was administered and when this was not enough to control the pain, the anesthetic technique was deemed as fail and switched to general anesthesia. 
Apart from VAS score recordings, patient's anthropometric data and times from the start of the sonographic examination until local anesthetic administration for the obturator nerve (phase 1) and the femoral and sciatic nerves (phase 2) were also included. Time for the whole anesthetic procedure, including local anesthetic administration and block assessment for the three nerves was also recorded. Data concerning the need for opioids and general anesthesia were also taken. BMI was calculated as weight in $\mathrm{kg}$ divided by the square of height in meters. VAS scores and block grades were treated as continuous variables. Data are presented as mean \pm standard deviation for numerical distributions and as percentages for categorical characteristics.

\section{Results}

In this 1 year study period, 226 patients were referred to our anesthesiology clinic for elective ACL reconstructive surgery and 57 of them were studied assigned in the two specialists of our study team. Demographic and somatometric data of our study population are presented in Table 1 in which more than half of these patients were athletes, including in majority soccer players and followed by basketball and water polo players, kick boxers and dancers. The sonographic recognition of obturator nerve was easy and quick in all cases. Excluding the preparation time, the time for the induction of obturator nerve block (Fig. 1, phase 1) is also shown in Table 1. Assessing this block with lifting-leaving down the leg produced satisfactory results in $24.0 \pm 5.07 \mathrm{~min}$ $(\min =18, \max =36)$ in all patients. Table 1 displays the cumula-

Table 1. Demographic, Somatometric and Intraoperative Data

\begin{tabular}{|c|c|}
\hline Number of patients & 57 \\
\hline Gender (Male) & $51 / 57(89.4 \%)$ \\
\hline Athletes & $35 / 57(61.4 \%)$ \\
\hline Age (yr) & $26.6 \pm 8.0(\max =49, \min =18)$ \\
\hline Weight (kg) & $76.7 \pm 9.9(\max =100.0, \min =55.0)$ \\
\hline Height (m) & $1.76 \pm 0.0(\max =1.91, \min =1.55)$ \\
\hline BMI & $24.6 \pm 2.8(\max =30.1, \min =18.6)$ \\
\hline Time for ON block (sec) & $119.9 \pm 79.2(\max =300, \min =32)$ \\
\hline Time for FS blocks (sec) & $324.8 \pm 252.7(\max =1269, \min =95)$ \\
\hline \multicolumn{2}{|l|}{ Block grade (n, pts) } \\
\hline $\mathrm{F}($ sensation $)>0$ & $0 / 57(0.0 \%)$ \\
\hline $\mathrm{F}$ (motor function) $>0$ & $1 / 57(1.7 \%)$ \\
\hline $\mathrm{S}($ sensation $)>0$ & $0 / 57(0.0 \%)$ \\
\hline $\mathrm{S}($ motor function $)>0$ & $8 / 57(14.0 \%)$ \\
\hline Tourniquet time (min) & $75.6 \pm 16.2(\max =105, \min =37)$ \\
\hline \multicolumn{2}{|l|}{ VAS > 0 (n, pts) } \\
\hline Tourniquet & $2 / 57(3.5 \%)$ \\
\hline Graft & $12 / 57(21.0 \%)$ \\
\hline Fentanyl $(\mu g)$ & $120.1 \pm 64.6(\max =250, \min =50)$ \\
\hline Midazolam (mg) & $1.86 \pm 0.8(\max =5.0, \min =1.0)$ \\
\hline Propofol (n, pts) & $6 / 57(10.5 \%)$ \\
\hline General anesthesia (n, pts) & $1 / 57(1.7 \%)$ \\
\hline
\end{tabular}

Data are expressed as means \pm SD. BMI: body mass index, ON: obturator nerve, F: femoral, S: sciatic, VAS: visual analogue scale. tive time for femoral-sciatic block (excluding preparation time) and the block grades for sensation and motor function for each after 30 minutes and the blocks were graded for sensation as 0 (zero) in all patients. The average grade for motor block for femoral nerve was $0.02 \pm 0.13$ and for sciatic nerve was $0.14 \pm 0.35$. One patient showed remaining motor function graded as 1 for the femoral block, and the motor block of the sciatic nerve was graded as one for 8 patients. Fentanyl and midazolam were administered in all patients and the mean total doses are shown in Table 1. Inflation of tourniquet resulted in VAS score of 3 and 5 in only 2 patients (3.5\%) and grafting induced VAS score $4.9 \pm 2.3(\min =1, \max =8)$ $(\mathrm{n}=12 ; 21.0 \%)$. Patients, who had VAS $>0$, received additional fentanyl intraoperatively. Six patients required additional propofol for further sedation $(118.3 \pm 70.5 \mathrm{mg}$; $\min =70$, $\max =260)$. After propofol administration, 3 patients were heavily sedated and were ventilated with a face mask for a short period of time. Furthermore, because of inadequate block, one of them required general anesthesia with positive pressure ventilation which was achieved after a laryngeal mask airway insertion. No patient complained for nausea or vomiting in the perioperative period. Finally, morphine was given for postoperative analgesia in 46 patients in a mean total daily dose of $8.6 \pm 5.8 \mathrm{mg}$.

\section{Discussion}

In the present study, we have demonstrated that ACL reconstructive surgery in young subjects with well-developed adductors can be performed under selective peripheral blockade of obturator, femoral and sciatic nerves with dual guidance. The sonographic recognition of obturator nerve and near-by structures were quick and easy and the identification of the correct position of neurostimulating needle was based on simultaneous contractions of adductor longus, brevis and magnus. For the assessment of block only motor function was evaluated with a simple and easily applicable clinical test. Incidentally, our study population included young athletic patients with large muscles, probably due to the fact that ACL tears and rupture are common in these subjects $[12,13]$. In our study most of the patients were male soccer players. This injury is usually quite painful and surgery may be inevitable, resulting in significant time lost from work and sports.

ACL reconstruction implicates tissue graft replacement of ACL to restore its function after injury. The hamstring tendon or the patellar tendon is usually harvested from the injured knee in order to be used as an autograft. Hamstring autografts are made with the semitendinosus tendon either alone or with gracilis tendon for a stronger graft. The tendon segments are folded and braided together to form a thick strand for the replacement graft, then the braided segment is threaded through the heads of tibia and femur and the ends are fixated with screws on the 
opposite sides of the two bones. The technique also requires an inflated thigh tourniquet. Most often these surgeries are performed under general anesthesia or central neural blockade and peripheral blocks are in supplementary use for postoperative analgesia. Presently, reports showing that this surgery can be done under obturator-femoral-sciatic block are very scarce. The lateral femoral cutaneous nerve does not have an important role during arthroscopic repair of anterior cruciate ligament and it does not have to be blocked separately.

The anatomy of obturator nerve shows a large degree of variability as confirmed by cadaveric studies. Leaving pelvis through the obturator canal, in the inguinal region, this relatively small nerve extends to the thigh dividing into anterior and posterior branches. In a significant number of individuals it divides early in the pelvis or during its course in the obturator canal or early after the level of the obturator foramen, following different and various branching patterns $[6,11]$. While dividing, the branches of common obturator nerve provide articular and cutaneous sensory nerves for the knee and the medial thigh. After performing an obturator nerve block, its sensory contribution to the structures of the knee is blocked, and this technique, as supplement of general anesthesia, provides a better quality of postoperative analgesia and recovery following knee surgery [3]. Moreover, obturator nerve block combined with other peripheral neural blockades allows ACL reconstructive surgery without the need of general anesthesia [4].

The sonographic visualization of obturator nerve may not to be easy due to the fact that it is small in size with a small deep course in the proximal thigh, and it lies between muscles [11]. Easy identifiable sonographic landmarks such as the femoral vessels are not very helpful and at times examiners use the pubic bone as a landmark [8] as the recognition of such landmarks may be of some value for the early stages of learning curve. Due to its anatomic variations, the obturator nerve itself may not be visible in ultrasonography at all. It is reported that the anterior and posterior rami are visible more often than the common nerve itself [9]. Many investigators recognize the muscles of the near area (pectineus, adductor longus and adductor brevis) and seek for echotexture resembling fascial connective tissue and fat. In cadavers, this nerve is surrounded by fascial layers [6]. In a study of 20 patients who received an obturator nerve block under dual guidance for postoperative pain management, a thick fascia was easily recognized in all of the patients. The nerve block was achieved after advancing the stimulating needle towards the fascia and maneuvering it to provoke muscle contractions [11]. Contrary to this, in a study of 22 patients the obturator nerve exhibited a hyperechoic flat or lip-shaped appearance with discrete internal hypoechoic dots [4] and it was suggested that this could be due to the fascial connective tissue, the fat and the aponeurotic tissue of the surrounding muscles. All these structures form a network where the nerve and its branches are embedded and, from the other hand, may facilitate the spread of the local anesthetic solution around them. Ultrasound guided interfascial injection of local anesthetic may lead to similar to obturator nerve block clinical results, without the need of nerve stimulation $[14,15]$.

In our study, the anterior and/or posterior divisions were easily recognized and tracing proximally ultrasound probe transducer revealed the "spider net" image. The image is formed by the fascial elements, the nerve itself, the sigmoid shaped ascending anterior division between adductor longus and adductor brevis muscles, the posterior division, and possibly by the hip articular ramus branching in a wide angle in respect to the course of the common nerve. The "spider net" image, although consistently reproduced, showed a notable variability, possibly due to the variations of the nerve and its branches as well as to variations in muscle volume, or due to the inter-examiners variability, after small changes in the position of the probe, preferred a certain view.

The depth in which obturator nerve lies depends on the somatometric data of the patient and on the degree of development and thickness of the surrounding structures as the muscles. In case of a block, the needle length on which obturator nerve or its branches can be found depends also on the angle of the needle with the skin. Introducing the needle with an out-of-plane approach may result to a different distance compared to an in-plane approach. In a feasibility study of ultrasound guided obturator nerve block, Fujiwara et al. [10] provided an image showing the skin to obturator nerve with a distance of less than $2 \mathrm{~mm}$, which was criticized by Anagnostopoulou et al. [16]. In a sonographic study by Soong et al. [9] the common obturator nerve, the anterior division and the posterior division were found at 25.9 $\mathrm{mm}, 15.5 \mathrm{~mm}$ and $29.3 \mathrm{~mm}$, respectively, distances not differing significantly from those reported by Manassero et al. [15]. Other report by Akkaya et al. [17] found that the sonographic values of the depth of the obturator nerve, its anterior branch and posterior branch were $32.4,28.2$, and $39.2 \mathrm{~mm}$, respectively. A skin to nerve distance of $30 \pm 7 \mathrm{~mm}$ was observed by Helayel et al. [4], while in Anagnostopoulou et al. [11], the sonographically measured distance of the fascia where the obturator nerve is embedded measured from the skin surface was $4.0 \pm 0.6 \mathrm{~cm}$. Although our study cannot provide exact information about the depth in which obturator nerve lies, our impression would have been approximately $2.5 \mathrm{~cm}$ since we approached the nerve just where adductor longus begins, before it gains its volume.

In our assessment method we examined only the motor block of the obturator nerve, since its sensory distribution is highly variable. The motor response of many blocks is usually assessed in a similar way in the everyday clinical practice. We assess the success of the block with a simple clinical approach, by ask- 
ing the patient to lift his leg. If the obturator block is successful the patient will not lift the leg directly upwards, but he will lift it diagonally and outwards and hold it there steadily, since the femoral nerve is still working. When the patient will be asked to leave down his leg, he will put it down laterally and will not be able to adduct it to medial line. The adductor brevis, adductor longus, adductor magnus, pectineus, and gracilis are all involved in adduction. In most of the subjects, pectineus is innervated by the femoral nerve and rarely does it accept a branch from the anterior division of the obturator nerve [11]. Nevertheless, in a successful obturator nerve block the unaffected pectineus is not enough to produce a hip adduction. In case of anterior branch block, the non functioning adductor longus and in part adductor brevis leave lifting and leaving down the leg almost unaffected, as adductor magnus is the big muscle that moves the leg inwards. Finally, in case of posterior branch block, adductors magnus and brevis are both not totally but in part nonfunctioning as the first is innervated by both the obturator and sciatic nerves and the second accepts a branch from the anterior division. As a result, lifting and leaving down the leg remains again unaffected. In this study the test was performed in the time-consuming way described only for the purposes of the study. In the everyday clinical practice the "lift-leave down" the leg test can be performed after all three blocks are performed, in a timely manner. Motor block assessment can be done by measuring adductor muscle strength with a pre-inflated blood pressure cuff between patient's knees as described by Helayel et al. [4] who reported a decrease of adductor strength from $147 \pm 29$ to $85 \pm 19 \mathrm{mmHg}(57.8 \%)$ at 30 minutes and a $91 \%$ success rate for blocking the obturator nerve, and a decrease of strength of $82.2 \%$ at 15 minutes was observed by Sinha et al. [14]. As the contribution of each adductor muscle in thigh adduction cannot be differentiated, the obstacle with this method is that if only the anterior or the posterior branch is blocked, a reduction in adductors strength is still measured.

In our study, we evaluated the feasibility of performing anterior cruciate ligament reconstruction exclusively under individual dual-guided blockade of obturator, femoral and sciatic nerves in young patients. Based on our results we propose that, in experienced hands, this method is safe and clinically feasible. Concerning the obturator nerve block, positioning the probe proximally to the inguinal crease below the level of the inguinal ligament decreases the possibility of falsely identifying branches instead of the main trunk of the obturator nerve. Moreover, when performing dual-guided block, it is mandatory that all the three muscles namely adductor longus, brevis and magnus are contracting. From our results we conclude also that the simple "lift-leave down" the leg test provides adequate assessment for the block and further studies are needed to determine the efficacy of this method in other kinds of knee surgery and in patients with different characteristics.

\section{References}

1. Auroy Y, Benhamou D, Bargues L, Ecoffey C, Falissard B, Mercier FJ, et al. Major complications of regional anesthesia in France: The SOS Regional Anesthesia Hotline Service. Anesthesiology 2002; 97: 1274-80.

2. Jankowski CJ, Hebl JR, Stuart MJ, Rock MG, Pagnano MW, Beighley CM, et al. A comparison of psoas compartment block and spinal and general anesthesia for outpatient knee arthroscopy. Anesth Analg 2003; 97: 1003-9.

3. Macalou D, Trueck S, Meuret P, Heck M, Vial F, Ouologuem S, et al. Postoperative analgesia after total knee replacement: the effect of an obturator nerve block added to the femoral 3-in-1 nerve block. Anesth Analg 2004; 99: 251-4.

4. Helayel PE, da Conceição DB, Pavei P, Knaesel JA, de Oliveira Filho GR. Ultrasound-guided obturator nerve block: a preliminary report of a case series. Reg Anesth Pain Med 2007; 32: 221-6.

5. Farny J, Drolet P, Girard M. Anatomy of the posterior approach to the lumbar plexus block. Can J Anaesth 1994; 41: 480-5.

6. Harvey G, Bell S. Obturator neuropathy. An anatomic perspective. Clin Orthop Relat Res 1999; 363: 203-11.

7. Bouaziz H, Vial F, Jochum D, Macalou D, Heck M, Meuret P, et al. An evaluation of the cutaneous distribution after obturator nerve block. Anesth Analg 2002; 94: 445-9.

8. Choquet O, Capdevila X, Bennourine K, Feugeas JL, Bringuier-Branchereau S, Manelli JC. A new inguinal approach for the obturator nerve block: anatomical and randomized clinical studies. Anesthesiology 2005; 103: 1238-45.

9. Soong J, Schafhalter-Zoppoth I, Gray AT. Sonographic imaging of the obturator nerve for regional block. Reg Anesth Pain Med 2007; 32: 146-51.

10. Fujiwara Y, Sato Y, Kitayama M, Shibata Y, Komatsu T, Hirota K. Obturator nerve block using ultrasound guidance. Anesth Analg 2007; 105: 888-9.

11. Anagnostopoulou S, Kostopanagiotou G, Paraskeuopoulos T, Chantzi C, Lolis E, Saranteas T. Anatomic variations of the obturator nerve in the inguinal region: implications in conventional and ultrasound regional anesthesia techniques. Reg Anesth Pain Med 2009; 34: 33-9.

12. Prodromos CC, Han Y, Rogowski J, Joyce B, Shi K. A meta-analysis of the incidence of anterior cruciate ligament tears as a function of gender, sport, and a knee injury-reduction regimen. Arthroscopy 2007; 23: 1320-5.e6. 
13. Waldän M, Hägglund M, Magnusson H, Ekstrand J. Anterior cruciate ligament injury in elite football: a prospective three-cohort study. Knee Surg Sports Traumatol Arthrosc 2011; 19: 11-9.

14. Sinha SK, Abrams JH, Houle TT, Weller RS. Ultrasound-guided obturator nerve block: an interfascial injection approach without nerve stimulation. Reg Anesth Pain Med 2009; 34: 261-4.

15. Manassero A, Bossolasco M, Ugues S, Palmisano S, De Bonis U, Coletta G. Ultrasound-guided obturator nerve block: interfascial injection versus a neurostimulation-assisted technique. Reg Anesth Pain Med 2012; 37: 67-71.

16. Anagnostopoulou S, Kostopanagiotou G, Paraskeuopoulos T, Alevizou A, Saranteas T. Obturator nerve block: from anatomy to ultrasound guidance. Anesth Analg 2008; 106: 350.

17. Akkaya T, Ozturk E, Comert A, Ates Y, Gumus H, Ozturk H, et al. Ultrasound-guided obturator nerve block: a sonoanatomic study of a new methodologic approach. Anesth Analg 2009; 108: 1037-41. 